\title{
Online Rail Straightness Measurement Based on Parallel Computing
}

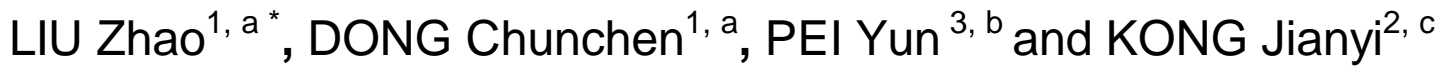

${ }^{1}$ College of Computer Science \& Technology, Wuhan University of Science and Technology, China

${ }^{2}$ College of Machinery and Automation, Wuhan University of Science and Technology, China

${ }^{3}$ Equipment Department, Wuhan Iron and Steel Group Corporation, China

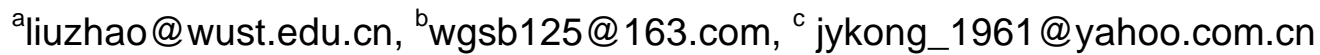

Keywords: rail; straightness measurement; online; parallel computing.

Abstract. Rail straightness measurement is critical in rail production, in which the detecting speed, accuracy and stability directly affect the overall rail production. In this paper, using modern technology such as laser sensors, data acquisition, and computing technology, an innovative rail straightness measurement method was designed and implemented based on parallel computing. In the design, 14 laser displacement sensors and 2 rotary encoders were used to measure respectively 7 level displacements, 7 vertical displacements, and rail transmission displacement in real-time. After those signals conversed to digital and transferred to server computer, CPUs and GPUs will: 1) synchronous matching, 2) inner block analyzing, 3) block analyzing, 4) frame analyzing, 5) straightness analyzing. The disturbing factors, such as iron oxides, side sliding, low frequency vibrations, and high frequency vibrations were and eliminated. Finally, get the rail straightness information and output, save, transfer, or used to guide production. Experiments and practical application show that, the method not only is feasible, but also it detection speed can up to $3 \mathrm{~m} / \mathrm{s}$, and accuracy up to $0.05 \mathrm{~mm}$.

\section{Introduction}

With the development of modern railway system and the increasingly fierce market competition, people are increasingly demanding high quality of the rail products, including: straightness, toughness, dimensions, smoothness, and so on. Rail straightness measurement is critical in rail production, in which the detecting speed, accuracy, and stability directly affect the overall rail production.

There are some rail straightness measurement products, but they have some kinds of problems when used in China, includes: 1) inspection speed less than $2 \mathrm{~m} / \mathrm{s}, 2$ ) too sensitive to iron oxides, 3) too sensitive to vibration, 4) too expensive.

As a production application, rail straightness measurement system functional specifications and requirements including: 1) Equipment size isn't longer than $3 \mathrm{~m}, 2$ ) Detection speed can up to $3 \mathrm{~m} / \mathrm{s}, 3$ ) Accuracy up to $0.05 \mathrm{~mm}$.

In this paper, in order to solve the above problems, an innovative rail straightness measurement method was proposed, in which using laser sensors to improve measure accuracy, using modern data acquisition modules to ensure real-time and synchronization, and using parallel computing and intelligent parallel algorithm to improve inspection speed.

\section{System architecture Design of the Rail Straightness Measurement}

The rail straightness measurement system is made up of 3 subsystems: 1) sense subsystem, 2) acquisition and synchronization subsystem, and 3) data analysis and information fusion subsystem.

Sense subsystem is made up of 7 top or vertical displacement laser sensors (T1to T7), 7 side or level displacement laser sensors (S1to S7), and 2 rotary encoders (A and B). The distance between every two adjacent vertical displacement sensors or every two adjacent level displacement sensors is $0.5 \mathrm{~m}$.

Acquisition and synchronization subsystem is made up of 2 modules: data acquisition module and data synchronization module.

Data analysis and information fusion subsystem is made up of 4 modules: 1) inner block analyzing module, 2) block analyzing module, 3) frame analyzing module, 4) straightness analyzing module. 
The System architecture is shown as Fig. 1.

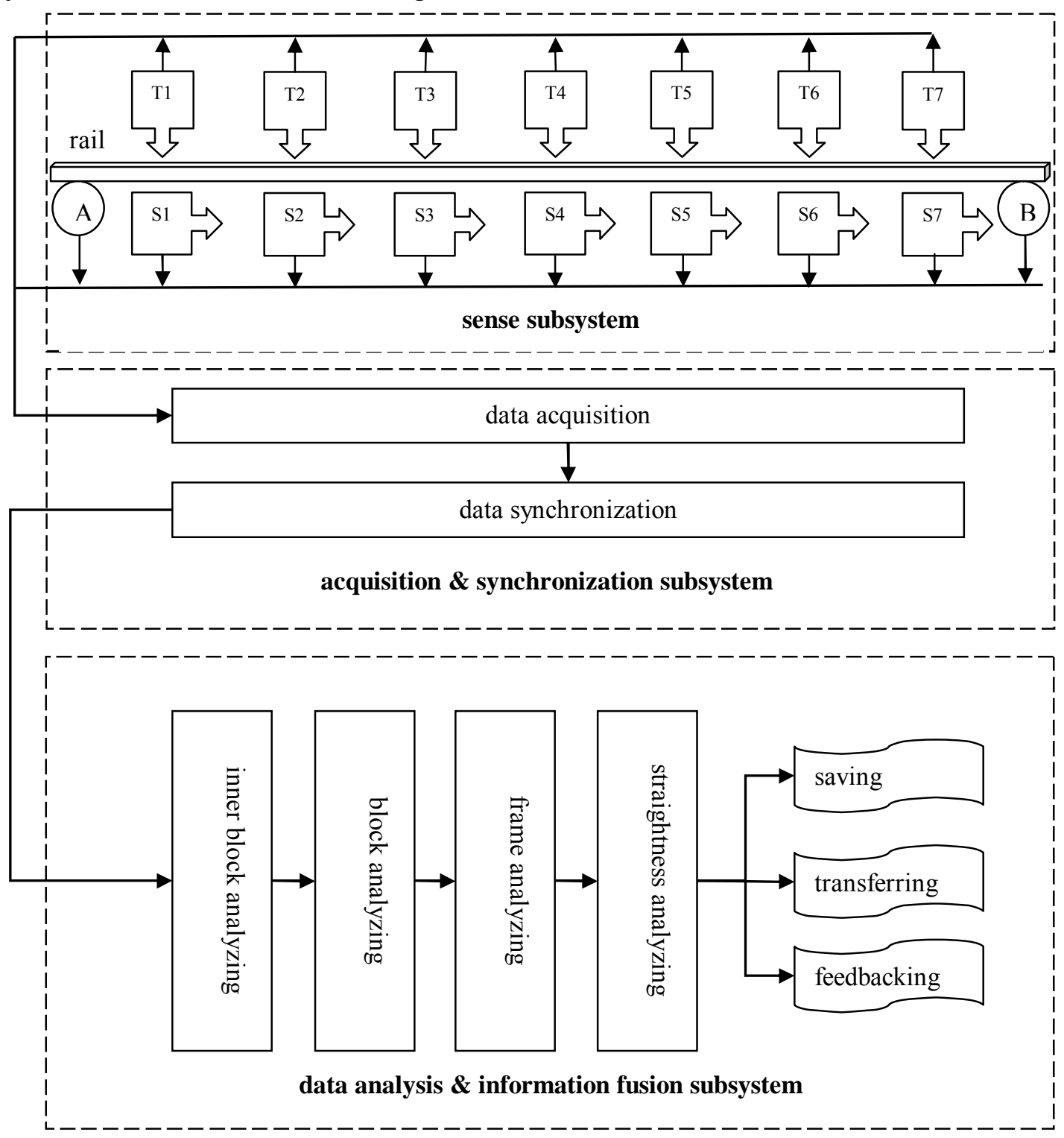

Figure 1. System architecture

\section{Online Rail Straightness Measurement Based on Parallel Computing}

Acquisition and Synchronization Based on Parallel Computing. By synchronous acquisition module to control and acquisition, in which each sensor set by maximum sampling frequency in time $\mathrm{t} 1$ for data acquisition, and then in time $\mathrm{t} 2$ only for data transmission. The paper designed the following intermittent synchronous acquisition method, shown as Fig. 2.

In order to shorten the acquisition and synchronization time of multichannel combined acquisition, a parallel acquisition algorithm is proposed.

1) Copy the periodic acquisition of data, 16 signal channels, to shared memory;

2) By analyze data from 2 rotary encoder channels, searching in 14 displacement data for $t 1, t 2$ corresponds data;

3) For 14 channels data correspond to the $\mathrm{t} 1, \mathrm{t} 2$, parallel computing: $\mathrm{y}=\mathrm{k} * \mathrm{x}+\mathrm{b}$. 


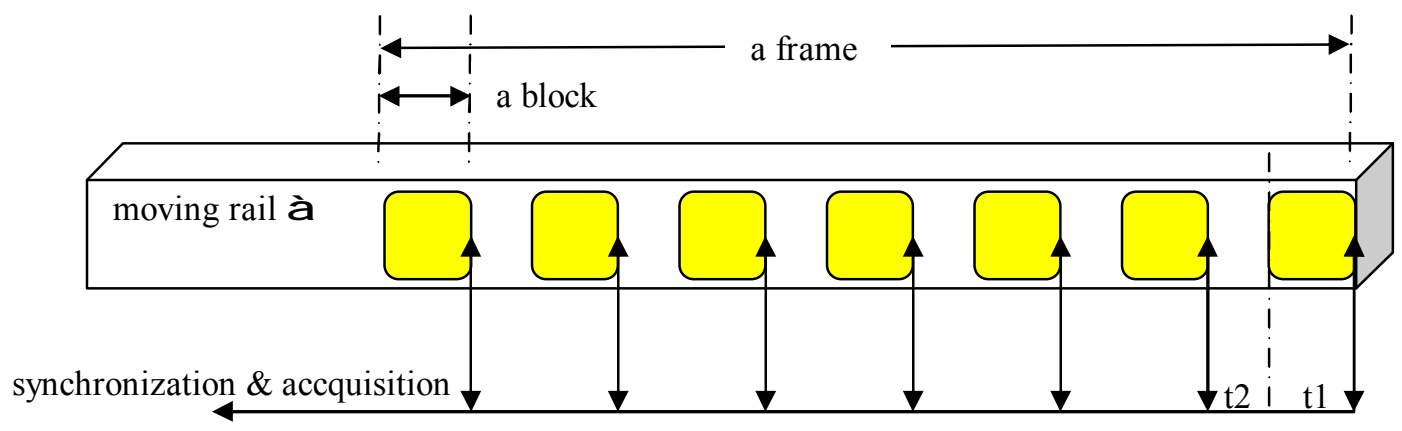

Figure 2. Block, Frame, and Synchronization

Inner block analyzing. Data within the block can be seen as a superposition of some data, including the roughness, iron oxides, side sliding. The purpose of inner block data analysis is to use the frequency domain, time domain characteristics of the data itself, disturbing data separation and, where possible, eliminated. Fig. 3 is a roughness and scale introduction of displacement can be mean and local mean elimination.

Figure 3. Block, Frame, and Synchronization

Block analyzing module. After synchronized, the data implied location information about rail of the time $\mathrm{t} 1$ in the measuring frame. Block data analyzing eliminate the impact resulting from side sliding, gets local straightness which mixed with low frequency vibration, while the low frequency vibration will be eliminated by frame analyzing.

Frame analyzing module. For each block corresponds to a small area of rail, multiple measurements at different time for the same small area, could eliminate low frequency vibration. And for that the repeated measurement over time may only occur in a different sensors, this paper propose analyze frame data, which contains 7 simultaneous data block. The task eliminates the effect of low frequency vibration, side sliding, linearity error, and random error.

Straightness analyzing module. According to measurement sampling precision in the rail transfer direction, 5 local flatness analyzing processes were designed to parallel execution, and then, fitting these 5 results online, updating the straightness of the whole current data.

Straightness valuing module. According to the current straightness data, calculating the baseline direction and baseline position for straightness valuing, and then, based on industry standards, valuing the flatness of the current interval. Fig. 4 is a picture of measuring and valuing online.

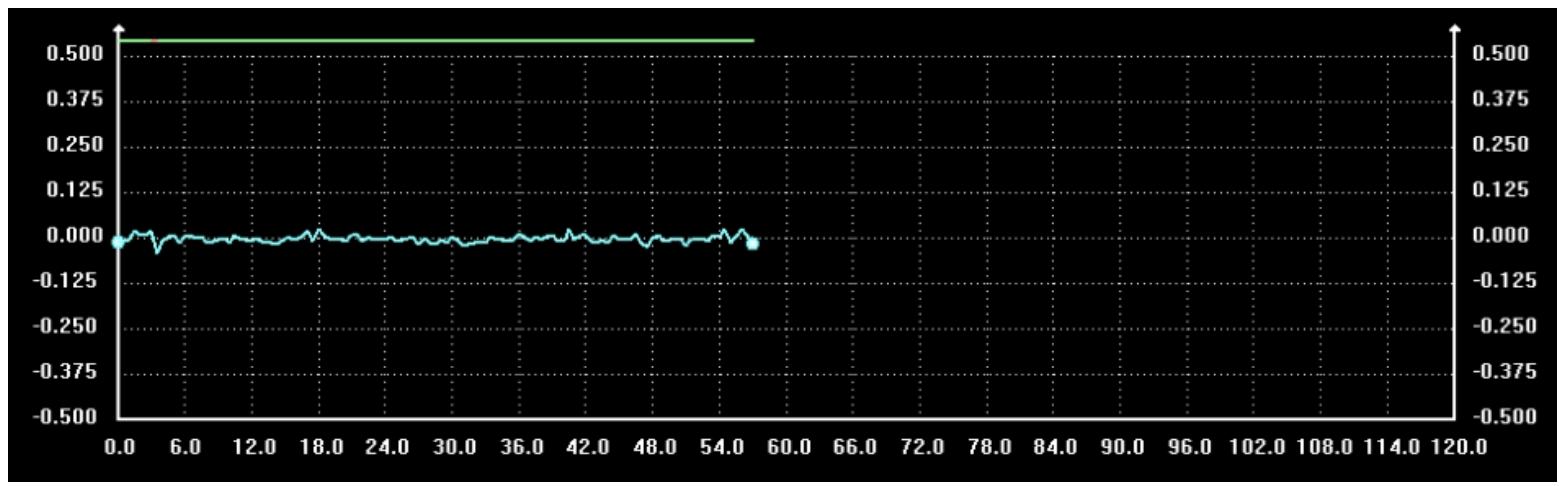

Figure 4. Rail straightness measuring and valuing online

\section{Summary}

The paper proposed an innovative rail straightness measurement method based on parallel computing, By means of synchronous matching, inner block analyzing, block analyzing, frame 
analyzing, and straightness analyzing, disadvantages, such as iron oxides, side sliding, low frequency vibrations, and high frequency vibrations were overcame. Tab. 1 shows straightness measurement experiment results, in which the stand rail are measured 50 times, and the mean square error of results. Experiments and practical application show that, the method not only is feasible, but also it detection speed can up to $3 \mathrm{~m} / \mathrm{s}$, and accuracy up to $0.05 \mathrm{~mm}$.

TABLE I. STRAIGHTNESS MEASUREMENT EXPERIMENT RESULTS

\begin{tabular}{|c|c|c|}
\hline Measure Items & MSE $(\mathbf{m m})$ & Max error $(\mathbf{m m})$ \\
\hline horizontal $0-1.5 \mathrm{~m}$ & 0.03 & 0.05 \\
\hline horizontal $1.5-2.0 \mathrm{~m}$ & 0.03 & 0.04 \\
\hline horizontal rail body & 0.05 & 0.06 \\
\hline vertical $0-1.5 \mathrm{~m}$ & 0.02 & 0.02 \\
\hline vertical $1.5-2.0 \mathrm{~m}$ & 0.02 & 0.02 \\
\hline vertical rail body & 0.02 & 0.02 \\
\hline
\end{tabular}

\section{Acknowledgements}

This work was supported by the National Natural Science Foundation of China (Grant No. $51174151)$.

\section{References}

[1] Kiyofumi Matsuda, Maitreyee Roy, Tomoaki Eiju, John W. O'Byrne, Colin J. R. Sheppard. Straightness measurements with a reflection confocal optical system--an experimental study. Applied Optics, vol. 41, no. 19, pp. 3966-3970, 2002.

[2] Cheng Weiming, Zhang Weina, Song Wei, Liu Liang, Sun Guiqing. Straightness measurement for long-length rails of bridge crane. International Conference on Measuring Technology and Mechatronics Automation - ICMTMA, 2011.

[3] Figliola, R. S., Beasley, D. E., Theory and Design for Mechanical Measurements, 3rd ed., J. Wiley \& Sons, New York, 2000.

[4] J Unkuri, A Rantanen, J Manninen, V-P Esala, A Lassila. Interferometric $30 \mathrm{~m}$ bench for calibrations of 1D scales and optical distance measuring instruments, Measurement Science \& Technology, vol. 23, no. 9, 2012.

[5] Jooho Hwang, Chun-Hong Park, Wei Gao, Seung-Woo Kim, A three-probe system for measuring the parallelism and straightness of a pair of rails for ultra-precision guideways, International Journal of Machine Tools \& Manufacture, vol. 47, no. 7, pp. 1053-1058, 2007

[6] S V Tchaban, Status and prospects of NDT for rail quality at Kuznetsk Steel Works, Insight, vol. 45, no. 6, pp. 421-423, 2003.

[7] Kiyono, S., Profile measurement using software datums, J. of JSPE, Vol. 61, No. 8, pp. 1059-1063, 1995.

[8] Gao, W., and Kiyono, S., High accuracy profile measurement of a machined surface by the combined method, Measurement, Vol. 19, No. 1, pp. 55-64, 1996.

[9] Fung, E. H. K., and Yang, S. M., An approach to on-machine motion error measurement of a linear slide, Measurement, Vol. 29, pp. 51-62, 2001.

[10] Kounosu, K., and Kishi, T., Measurement of surface profile using smoothed serial three point method, J. of JSPE, Vol. 61, No. 5, pp. 641-645, 1995. 